\title{
Optimization of a Lunar Pallet Lander Reinforcement Structure using a Genetic Algorithm
}

Adam Burt, NASA Marshall Space Flight Center, Space Systems Department/ES22, Huntsville, AL 35812

\section{Introduction}

In this paper, a unique system level spacecraft design optimization will be presented. A Genetic Algorithm is used to design the global pattern of the reinforcing structure, while a gradient routine is used to adequately stiffen the sub-structure. The system level structural design includes determining the optimal physical location (and number) of reinforcing beams of a lunar pallet lander deck structure. Design of the substructure includes determining placement of secondary stiffeners and the number of rivets required for assembly. . In this optimization, several considerations are taken into account. The primary objective was to raise the primary natural frequencies of the structure such that the Pallet Lander primary structure does not significantly couple with the launch vehicle. A secondary objective is to determine how to properly stiffen the reinforcing beams so that the beam web resists the shear buckling load imparted by the spacecraft components mounted to the pallet lander deck during launch and landing. A third objective is that the calculated stress does not exceed the allowable strength of the material. These design requirements must be met while, minimizing the overall mass of the spacecraft. The final paper will discuss how the optimization was implemented as well as the results. While driven by optimization algorithms, the primary purpose of this effort was to demonstrate the capability of genetic algorithms to enable design automation in the preliminary design cycle. By developing a routine that can automatically generate designs through the use of Finite Element Analysis, considerable design efficiencies, both in time and overall product, can be obtained over more traditional brute force design methods.

\section{Mission Description}

The pallet lander design concept is being developed to carry a rover to the lunar surface. The current concept involves utilizing a thin, flat deck allowing the proposed rover to simply roll off onto the surface. Therefore, a stiffened structure concept is needed to reinforce the top surface skin and carry the loads imparted by the rover and other spacecraft components such as propellant tanks and navigation equipment, while maintaining a minimal amount of structural mass. The starting design is shown in Figure 1. Using this initial design space and

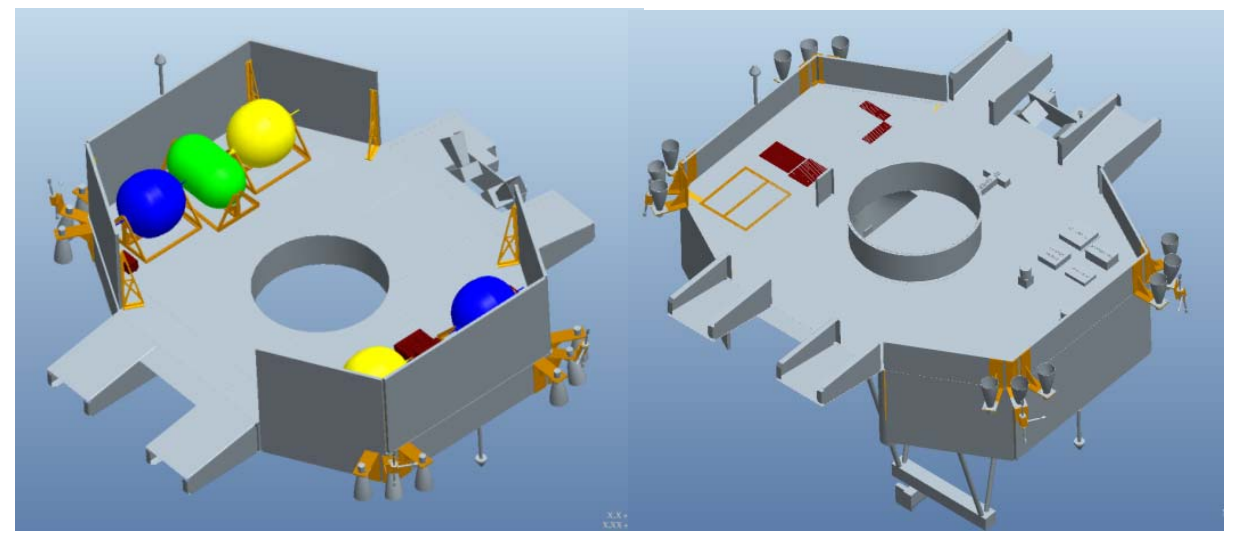

Figure 1: Lunar Pallet Lander Initial Design Configuration Left image is top surface skin where rover will be placed. The right image shows underneath the deck where reinforcement is required. 
manufacturing constraints discussed in the Design Space Summary, a genetic algorithm was used to determine the most efficient way to reinforce the structure.

\section{Design Space Summary}

The optimization was implemented with several manufacturing constraints taken into account which helped to reduce the final required design space. First, it was decided up front that the pallet lander would be fabricated using sheet metal. This decision was made to reduce cost and based on initial sizing that showed that very small thicknesses were able to carry the shear loading. The reinforcing beams would be a constant channel cross-section where possible to, again, reduce cost. The final assumption would be that the reinforcing pattern produced would be rectangular pockets only. All of these constraints were utilized during the optimization.

\section{Optimization Process Summary}

Several exploratory optimization analyses (not discussed in detail here), showed that a rectangular reinforcing pattern had the most promise, but that uniform pockets such as that seen in an orthogrid type design would not provide sufficient stiffness to meet the natural frequency requirements. Therefore the final optimization allowed for non-uniform rectangular pockets to be generated. To do this a script was written to automatically generate a 2D Finite Element Model (FEM). The number of beams used and their location on the deck relative to each other were parameterized as the design space for the Genetic Algorithm. Once this model was generated, a modal analysis and linear static analysis were performed to obtain the results needed to develop an objective function. For the modal analysis, the modes with the highest modal effective mass were determined and considered to be the primary natural frequencies of the structure. Next, the stress results were assessed to ensure that the Von Misses stress in any element in the model was not found to exceed the allowable strength of the material. Finally the mass of the solution was assessed. This formed the objective function, taking on the form:

$$
\begin{gathered}
\text { obj }=\min \frac{\text { mass }}{W x+W y+W z} \\
\sigma_{\text {vonmisses }}<\frac{\text { Ultimate Strength }}{\text { Factor of Safety }}
\end{gathered}
$$

Where Wx, Wy and Wz represent the natural frequency of the modes that have the highest modal effective mass for each translational direction.

This objective function will act to simultaneously minimize the mass while maximizing the natural frequency and ensuring that the stress must remain below the allowable strength of the material.

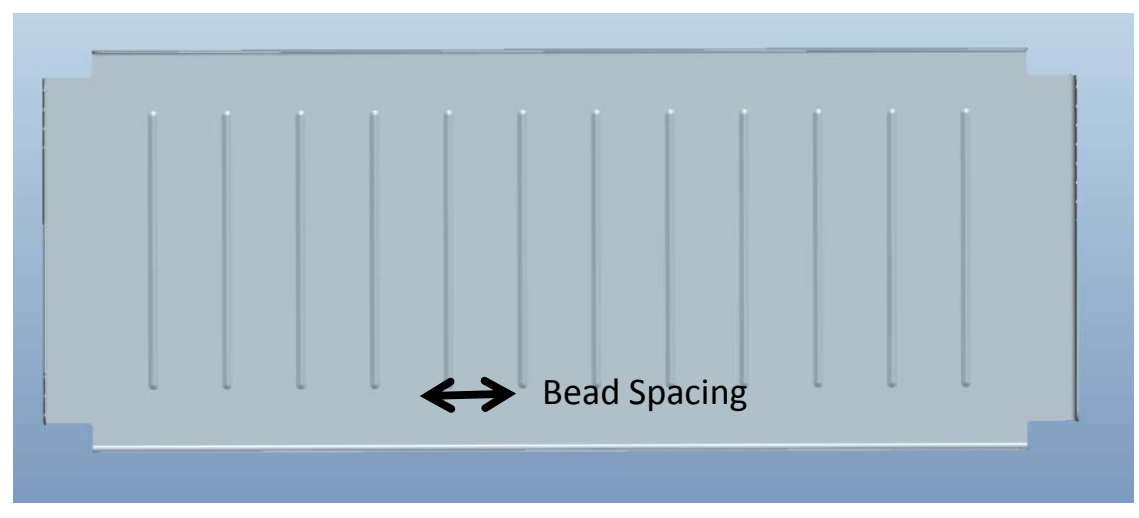

Figure 2: Sheet Metal Panels with Formed Beads Additional Physical Models 
Current work is focused on adding additional physical models to the optimization to increase the fidelity of the modeling results. First, the beams used to reinforce the deck must be assessed for buckling due to the shear load imparted onto the beam. With this shear load the optimizer will then determine how to adequately reinforce the beams themselves using either formed beads (and the minimum required bead spacing shown in Figure 2) or angle stiffeners riveted to the sheet metal beam web. The beaded and stiffened sheet metal is represented using Orthotropic Plate Theory, as is common with stiffened structures. This theory will be used to assess the plate's resistance to shear buckling and also the effect of the additional stiffness on the natural frequency of the overall structure. This process is completed for each beam. Next the shear flow can be determined for each beam and subsequently design the riveted joint required to carry the load. Therefore the number of required rivets can be determined and will be added to mass term used in the optimization. This sub-level optimization is performed for every solution produced by the genetic algorithm, thus the final optimization will be acting to minimize the number of rivets in the model as well as maximize the stiffness of the sub-structure to increase the primary natural frequencies.

\section{Results}

The results of the preliminary optimization yielded a solution with rectangular pockets that met the natural frequency and strength requirements. The optimization was driven using Altair's Hyperstudy that provides a genetic algorithm and post processing capability. The default setting for the Genetic Algorithm was used. Figure 3 shows how the natural frequency progressed with the evolution of the Pallet Lander and Figure 4 shows the final solution that was produced by the optimization.

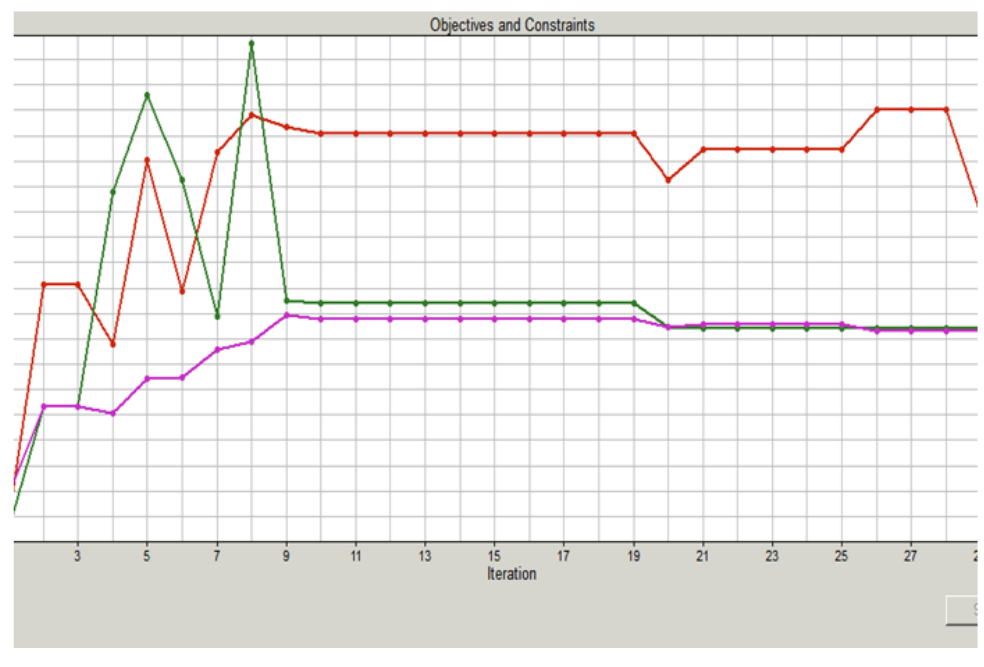

Figure 3: Evolution of the Primary Natural Frequency of the Structure

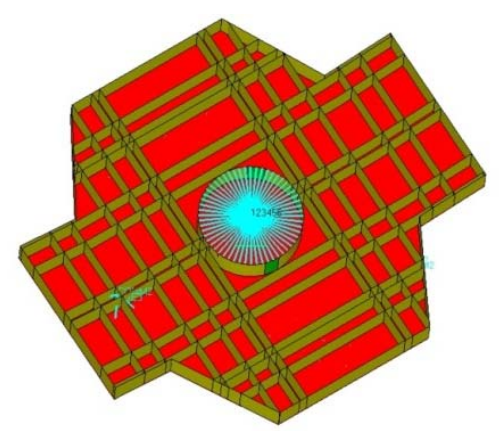

Figure 4: Optimal Reinforcement Design Produced by the Genetic Algorithm 


\section{References}

SanSoucie, M. P., Hull, P., Irwin, R., Tinker, M. L., \& Patton, B. (2005). Trade Studies for a Manned High-Power Nuclear Electric Propulsion Vehicle. 1st Space Exploration Conference: Continuing the Voyage of Discovery. Orlando, FL: AIAA.

Schnell, A., Tinker, M., Dozier, G., P., H., Alverson, L., A., G., et al. (2008). Development of XTOOLSS Preliminary Design of Space Systems Using Evolutionary Computation. 4th AIAA Multidisciplinary Design Optimization Specialist Conference. Schaumburg, IL.

Tinker, M., Steincamp, J., Stewart, E., Patton, B., Pannell, W., Newby, R., et al. (2004). Nuclear Electric Vehicle Optimization Toolset (NEVOT). 10th AIAA/ISSMO Multidisciplinary Analysis and Optimization Conference. Albany, NY.

Bruhn EF. Analysis and design of flight vehicle structures, Tri-state offset company. vol. 31965. 